\title{
Open science questions and missing measurements in the radiation belts of Jupiter
}

\author{
A White Paper to the National Research Council Planetary Science and Astrobiology Decadal
} Survey 2023-2032

Primary author: Quentin Nénon, Space Sciences Laboratory, University of California at Berkeley, nenon@berkeley.edu, +1 5102218868

Co-authors: G. Clark ${ }^{2}$, I. Jun ${ }^{1}$, P. Kollmann 2 , L. Liuzzo 3 , B. Mauk ${ }^{2}$, T. A. Nordheim ${ }^{1}$, A. R. Poppe $^{3}$, E. Roussos ${ }^{5}$, Y. Y. Shprits ${ }^{19,21}$, D. L. Turner ${ }^{2}$, E. E. Woodfield ${ }^{40}$

Endorsers: P. Addison ${ }^{28}$, O. Allanson ${ }^{22}$, L. Alves ${ }^{14}$, N. André ${ }^{31}$, L. Arruda ${ }^{49}$, B. Bertucci ${ }^{32}$, S. Bourdarie $^{7}$, P. Brandt ${ }^{2}$, G. Branduardi-Raymont ${ }^{33}$, B. R. Breer ${ }^{28}$, A. Brunet ${ }^{7}$, E. Bunce ${ }^{44}$, X. $\mathrm{Cao}^{13}$, C. Cohen ${ }^{52}$, I. Cohen ${ }^{2}$, J. F. Cooper ${ }^{12}$, I.A. Daglis ${ }^{27}$, I. Dandouras ${ }^{31}$, L. Da Silva ${ }^{14,26}$, I. de Pater $^{3}$, A. M. de Souza Franco ${ }^{14}$, R. T. Desai ${ }^{6}$, K. Dialynas ${ }^{9}$, A. Drozdov ${ }^{41}$, E. Echer ${ }^{14}$, S. Fatemi $^{10,43}$, Y. Futaana ${ }^{10}$, H. B. Garrett ${ }^{1}$, M. Gkioulidou ${ }^{2}$, P. Gonçalves ${ }^{48,49}$, Y. Hao $^{24}$, M. M. Hedman $^{45}$, R. B. Horne ${ }^{40}$, G. Hospodarsky ${ }^{13}$, H. Huybrighs ${ }^{25}$, C. M. Jackman ${ }^{46}$, X. Jia ${ }^{42}$, G. Jones $^{33}$, J. T. Keane ${ }^{1}$, K. K. Khurana ${ }^{41}$, H. Kita ${ }^{34}$, A. Kotova ${ }^{31}$, E. A. Kronberg ${ }^{23}$, N. Krupp ${ }^{5}$, S. Lejosne $^{3}$, W. Li ${ }^{11}$, X. Li ${ }^{50}$, V. Maget ${ }^{7}$, J. P. Marchezi ${ }^{14}$, C. Moeckel ${ }^{3}$, G. Murakami ${ }^{20}$, C. Paranicas $^{2}$, C. Paty ${ }^{51}$, M. Pinto ${ }^{49}$, C. Plainaki ${ }^{36}$, J. Rae ${ }^{33}$, L. H. Regoli ${ }^{2}$, O. Santolik ${ }^{37,38}$, D. Santos-Costa $^{8}$, T. Sarris ${ }^{39}$, Q. Q. Shi ${ }^{47}$, A. Sicard ${ }^{7}$, S. Simon ${ }^{28}$, H. T. Smith ${ }^{2}$, A. H. Sulaiman ${ }^{13}$, Y. Sun ${ }^{24}$, J. R. Szalay ${ }^{15}$, L. E. A. Vieira ${ }^{14}$, D. Wang ${ }^{19}$, Y. Wei ${ }^{29}$, P. K. G. Williams ${ }^{16,17}$, X. Wu ${ }^{30}$, Z. H. $\mathrm{Yao}^{30}$, C. Yuan ${ }^{29}$, P. Zarka ${ }^{35}$, Q. Zong ${ }^{24}$

${ }^{1}$ NASA Jet Propulsion Laboratory, Pasadena, CA, USA ${ }^{2}$ John Hopkins University/Applied Physics Laboratory, Laurel, MD, USA ${ }^{3}$ UC Berkeley, Berkeley, CA, USA ${ }^{4}$ LPAP, University of Liege, Liege, Belgium ${ }^{5}$ Max Planck Institute for Solar System Research, Göttingen, Germany ${ }^{6}$ Imperial College London, London, UK ${ }^{7}$ ONERA, Toulouse, France ${ }^{8}$ SwRI, San Antonio, TX, USA ${ }^{9}$ Academy of Athens, Athens, Greece ${ }^{10}$ Swedish Institute of Space Physics (IRF), Kiruna, Sweden ${ }^{11}$ Center for Space Physics, Boston University, Boston, Massachusetts, USA ${ }^{12}$ NASA Goddard Space Flight Center, Greenbelt, MD, USA ${ }^{13}$ University of Iowa, Iowa City, IA, USA ${ }^{14}$ Instituto Nacional de Pesquisas Espaciais, Sao Jose dos Campos, Brazil ${ }^{15}$ Princeton University, Princeton, NJ, USA ${ }^{16}$ Center for Astrophysics | Harvard \& Smithsonian, Cambridge, MA, USA ${ }^{17}$ American Astronomical Society, Washington, DC, USA ${ }^{19}$ German Research Center for Geoscience, GFZ-Potsdam, Potsdam, Germany ${ }^{20}$ JAXA/ISAS, Sagamihara, Kanagawa, Japan ${ }^{21}$ Institute for Physics and Astrophysics, University of Potsdam, Potsdam, Germany ${ }^{22}$ University of Reading, UK ${ }^{23}$ Ludwig Maximilian University of Munich, Munich, Germany ${ }^{24}$ Peking university, Peking, China ${ }^{25}$ ESA/ESTEC, Noordwijk, Netherlands ${ }^{26}$ State Key Laboratory of Space Weather, National Space Science Center, Chinese Academy of Sciences, China ${ }^{27}$ National and Kapodistrian University of Athens, Athens, Greece ${ }^{28}$ Georgia Institute of Technology, Atlanta, GA, USA ${ }^{29}$ Institute of Geology and Geophysics, Chinese Academy of Sciences, Beijing, China, ${ }^{30}$ University of Geneva, Geneva, Switzerland, ${ }^{\mathbf{3 1}}$ Institut de Recherche en Astrophysique et Planétologie, CNRS, CNES, Université de Toulouse, Toulouse, France ${ }^{32}$ University of Perugia, Perugia, Italy ${ }^{33}$ UCL Mullard Space Science Laboratory, Dorking, UK ${ }^{34}$ Tohoku Institute of Technology, Sendai, Japan ${ }^{35}$ LESIA, CNRS, Observatoire de Paris, PSL, Meudon, France ${ }^{36}$ Agenzia Spaziale Italiana (ASI), Rome, Italy ${ }^{37}$ Institute of Atmospheric Physics of the Czech Academy of Sciences, Prague, Czech Republic ${ }^{38}$ Faculty of Mathematics and Physics, Charles University, Prague, Czech Republic ${ }^{39}$ Democritus University of Thrace, Xanthi, Greece ${ }^{40}$ British Antarctic Survey, Cambridge, UK ${ }^{41}$ UCLA, Los Angeles, CA, USA $^{42}$ University of Michigan, Ann Arbor, MI, USA ${ }^{43}$ Department of physics at Umeå University, Umeå, Sweden ${ }^{44}$ University of Leicester, Leicester, UK ${ }^{45}$ University of Idaho, Moscow, ID, USA ${ }^{46}$ Dublin Institute for Advanced Studies, Dublin, Ireland ${ }^{47}$ Space Science Institute, School of Space Science and Physics, Shandong University, Shandong, China ${ }^{48}$ Lisbon University, Lisbon, Portugal ${ }^{49}$ LIP, Lisbon, Portugal ${ }^{50}$ LASP and AES, University of Colorado, Boulder, CO, USA ${ }^{51}$ University of Oregon, Eugene, OR, USA ${ }^{52}$ California Institute of Technology, Pasadena, CA, USA 
All sufficiently magnetized planets in the Solar System (the Earth, Jupiter, Saturn, Uranus and Neptune) are surrounded by extended torus-shaped regions filled with energetic electrons, protons, and heavier ions: the radiation belts. Radiation belts host various processes that accelerate charged particles from low to relativistic energies in a small enough region and over time scales that can be fully monitored in situ by space missions, unlike the acceleration sites of astrophysical particles. Radiation belts therefore offer the opportunity to perform ground truth measurements for a variety of high-energy physical processes and their synergies that operate throughout the universe. The radiation belts of Jupiter share several similarities with their counterparts at other planets and their investigation therefore enables the development of scalability laws that are necessary to probe similar, distant astrophysical systems ${ }^{1}$. At the same time, they exhibit significant differences that are detailed hereafter, so that they are key to our understanding of how these astrophysical objects work ${ }^{2,3,4,5}$. An added value of studying energetic charged particles at Jupiter is that they are 'messengers' which keep track of their interactions with the exotic and unique environment of the planet's inner magnetosphere: faint dust rings, small airless inner moons, the volcanic moon Io, the icy Europa which may host life, Ganymede with its own intrinsic magnetic field, and the inert Callisto. This way, the observation and modeling of radiation belts around giant planets has been shown to address major planetary science questions like characterizing planetary exospheres, or discovering previously unknown rings and moon-generated neutral torii ${ }^{6,8,9,10}$. The reverse path, i.e., the effect of radiation belt particles on planetary constituents (e.g., weathering of satellite surfaces) is also decisive for planetary science ${ }^{11,12,13}$.

Since $1959^{14}$, Earth-based observatories and space missions have constantly pushed forward and revolutionized the characterization and phenomenological understanding of Jupiter's radiation belts. This observational legacy will be further expanded by ongoing and planned missions to Jupiter, which will tremendously deepen our understanding of this complex system. Yet, in order to achieve understanding beyond characterization, critical measurements are missing. In this White Paper, we build upon the state of the art and the anticipated scientific developments on Jupiter's radiation belts to elaborate seven overarching scientific questions, highlight their importance for space physics, astrophysics and planetary science, and identify the required measurements that would enable their answers.

Required measurements include (1) the monitoring of the solar wind upstream of Jupiter, (2) the in-situ mapping of the charged particle distribution functions (electrons up to $\sim 100 \mathrm{MeV}$, ions into the $\mathrm{GeV}$ range) along with their background electromagnetic fields and waves environment in the magnetosphere and the radiation belts and (3) global context from remote sensing of the Jovian system in radio, UV, X-Rays and Energetic Neutral Atoms. All measurements should be performed simultaneously and achieve the maximum spatial coverage of Jupiter's radiation belts, including their core where extreme radiation levels are observed.

A space mission aiming to bring closure to these questions through such measurements would face unprecedented technological challenges in both instrumentation and mission design (see specific recommendations hereafter and in Roussos et al., 2019, submitted to the European Space Agency's Voyage 2050 call). Resolving the energy and directional distribution of differential charged particle intensity up to $\sim 1 \mathrm{GeV}$ is one of the key required measurements that is achieved in space observatories that observe high energy but low intensity cosmic rays ${ }^{15,16}$. Miniaturization for a mass-constrained planetary mission is feasible ${ }^{16}$ but still remains to be implemented. In addition, while crossing repeatedly through the planet's radiation belts is a viable technological feat, a mission diving in them deeper and longer than ever before raises the bar for radiation mitigation strategies even higher. A mission overcoming such challenges would not only answer the listed questions, but would also bring unanticipated disruptive 
discoveries, as occurred and will occur with Cassini at Saturn ${ }^{17}$, the Van Allen Probes at Earth ${ }^{18}$, and ongoing and future missions at Jupiter ${ }^{19,20}$.

The exploration of giant planet radiation belts by NASA has been, is now and will be conducted by the Planetary Science Division (PSD) of the Science Mission Directorate (SMD) with the Voyager, Galileo, Juno and Europa-Clipper missions. PSD also partners with the European Space Agency on instrumentation for the JUICE mission. The open science questions that are listed hereafter encompass the Jovian counterpart of the scientific objectives of the Van Allen Probes mission in the terrestrial radiation belts ${ }^{18}$, which was managed by the Heliophysics Division of SMD. In addition, brown dwarfs with Jovian-like magnetospheres ${ }^{21,22}$ and extrasolar planets are likely to possess radiation belts ${ }^{23}$, making the study of the Jovian radiation belts ground truth relevant to astrophysical investigations. Instrumentation development and future space missions dedicated in part or entirely to the radiation belts of Jupiter could therefore rely on a joint effort between the Planetary Science, Heliophysics, and Astrophysics divisions of SMD.

The last decadal survey ${ }^{24}$ states the importance of the radiation belts for understanding the exogenic weathering of the Jovian moons (question 6 in this White Paper). However, weathering cannot be studied in isolation from the physics of the radiation belts that drive it. In addition, questions on the physics of the Jovian radiation belts listed here are important on their own right, as explained earlier, but were not mentioned in the last decadal survey despite being aligned with the latest report of the Outer Planet Assessment Group ${ }^{7}$, which highlights the following overarching question: "How are planetary radiation belts formed and maintained?" The benefits of answering the listed questions for radiation belt physics, space physics, astrophysics, and planetary science in the Solar System and beyond are such that they should be given a high priority in the decadal survey. In addition, we recommend NASA and NSF to:

- Support an advanced study for a Van Allen Probes type, multi-spacecraft mission at Jupiter, with a design and instrumentation adjusted to the unique and extreme environment properties of the planet's radiation belts. Multi-spacecraft missions are a modern standard for space physics in the Solar System (Earth, the Moon, Mercury, Mars) as they enable to distinguish the different physical mechanisms at work with minimum ambiguity;

- Support the development of high-energy (1-1000s MeV) charged particle instruments able to provide full sky direction resolved observations with high energy resolution and advanced mass discrimination, and yet fit into the mass constraints and magnetic cleanliness requirements of a planetary mission;

- Consider any future mission targeted towards the Jovian system as an opportunity to advance radiation belt physics with dedicated energetic particle instruments. A potential mission to $\mathrm{Io}^{25}$ is an example of such an opportunity;

- Maintain and enhance regular campaigns of remote observations of the Jovian radiation belts (synchrotron radiation at radio wavelengths and X-rays).

\section{1) How are electrons accelerated and transported in the radiation belts of Jupiter?}

A common problem in space physics, from analyzing solar wind to cosmic rays, is how the energetic particles we measure were accelerated to the observed energies. The magnetosphere of Jupiter is the most powerful planetary electron accelerator, as $>70 \mathrm{MeV}$ electrons $(99.999 \%$ the speed of light) are observed ${ }^{26}$. While Earth's magnetosphere only accelerates electrons to several MeV during very extreme events, Jupiter does this all the time, making it the ideal laboratory to study particle acceleration. From remote observations of electron synchrotron emissions and from in situ data gathered by the Galileo mission, two distinct families of qualitative physical mechanisms have been proposed to explain electron acceleration at Jupiter. The first one is the radial and adiabatic transport of electrons from regions of low to higher 
magnetic field amplitude, that may be driven by interchange events, radial diffusion and/or convection (Figure 1). The second is the local non-adiabatic acceleration of electrons by gyroresonant interaction with electromagnetic waves, which can also contribute to particle losses. Whistler-mode chorus waves are a likely effective acceleration mechanism near Europa's orbit $^{27,28,29}$, as at Earth ${ }^{30,31}$ and maybe Saturn ${ }^{32}$. Figure 1 shows that the regions in which different physical processes can occur overlap in space and electron energy, in particular between $\mathrm{L}=6$ and $\mathrm{L}=15$ where electromagnetic waves may have an important role. Outside of this region, however, acceleration mechanisms may be unmixed, unlike at Earth, so that Jupiter would be an ideal testbed. As we cannot reach any quantitative conclusion even on the order of magnitude of these processes as a result of missing measurements, it is largely unknown if one is dominant over the others or if they act in unison, and whether we have yet identified all mechanisms and their roles for electron acceleration, transport and loss at Jupiter.

Figure 1. A summary of
possible processes that
may contribute to electron
transport, acceleration,
and losses in Jupiter's
radiation belts as a
function of L-shell
(magnetic equatorial
distance) and energy.

Missing in situ measurements: Most of the electron physics is observed at kinetic energies up to the low

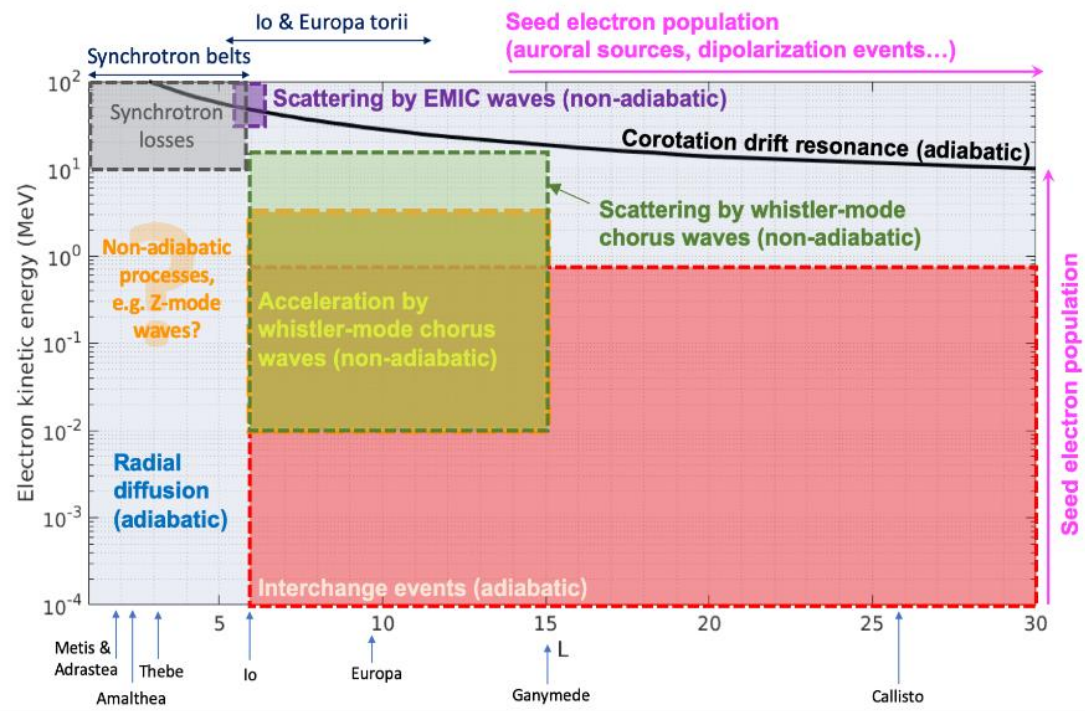
$\mathrm{MeV}$ range at Earth and Saturn, whereas it is shifted to higher energies at Jupiter; for instance, the radial transport of electrons with stationary guiding centers around Jupiter occurs at energies greater than tens of $\mathrm{MeV}$ (corotation drift resonance on Figure $1^{33}$ ). Direction and energy resolved observations of electrons at kinetic energies up to $\sim 100 \mathrm{MeV}$ are therefore necessary to capture their acceleration and loss mechanisms at Jupiter. These observations are needed throughout the radiation belts, with a particular emphasis on low- to mid-latitudes inward of Europa's orbit, which have not been and are not currently planned to be explored by an orbiting spacecraft. Complementary parameters that regulate electron acceleration and loss should be measured, such as cold plasma moments, and multicomponent measurements of the electromagnetic field and its variations, providing us not only with wave amplitudes but also with the polarization and propagation properties at frequencies lower than the local electron gyrofrequency.

\section{2) Cosmic Ray Albedo Neutron Decay: a universal source of radiation belt protons?}

The acceleration of particles to high energies can also happen outside of the magnetosphere of Jupiter, as occurs for GeV Galactic Cosmic Ray (GCR) ions. An efficient way to convert these ions into radiation belt particles is via the Cosmic Ray Albedo Neutron Decay process (CRAND): nuclear collisions of GCR ions with material targets (moons, rings, atmosphere) produce neutrons which then $\beta$-decay within the magnetosphere into several products, including a proton which inherits most of the energy from the parent neutron, and an electron. CRAND has been shown to be an effective supply of $>10 \mathrm{MeV}$ protons to the radiation belts of Earth ${ }^{34}$ and Saturn ${ }^{17}$ and may be important at any planet with a magnetic field sufficient to trap its products. It is therefore important to understand how this process scales with the parameters of 
a planetary system.

Jupiter's parameter regime is drastically different than at Earth and Saturn, so that Jupiter is an ideal steppingstone to understand how the CRAND coupling between planets and galaxies works. In particular, the planetary magnetic field is much more intense at Jupiter than at the other planets. It is unknown if this reduces the efficiency of CRAND, as fewer GCR ions are able to reach the atmosphere of Jupiter, or if it instead increases it: the large size of the Jovian magnetosphere enables to catch more decaying neutrons than at other planets, and the strong magnetic field amplitude close to the planet enables a slow but stable accumulation of energetic protons at energies higher than at other planets. The accumulation of CRAND protons at Saturn is balanced by the absorption effect of moons ${ }^{35}$. This process is drastically different at Jupiter as a result of the tilt between the planet's magnetic dipole and rotation axis ${ }^{36}$.

Missing in situ measurements: Direction and energy resolved observations of protons with kinetic energies higher than 10 s of $\mathrm{MeV}$ and up to several $\mathrm{GeV}$ would undoubtedly answer the question of the effectiveness of CRAND at Jupiter. CRAND fluxes would be the strongest inward of Io's orbit, where the magnetic field amplitude and proton trapping limit are at maximum. Measurements in this region would be comparable to those obtained by Cassini during the final ring grazing orbits near Saturn ${ }^{17}$.

\section{3) What is the origin of Jupiter's heavy ion radiation belts?}

The ion radiation belts of Jupiter are unique as the ratio of heavy ions to protons is much higher than that encountered at Earth and Saturn, with in particular abundant energetic sulfur and oxygen ions found from very close to Jupiter ${ }^{19}$ to the outermost part of the magnetosphere ${ }^{37}$. The ion composition makes the volcanic moon Io a source candidate of choice, but several important mysteries remain: how are cold plasma heavy ions accelerated to radiation belt energies higher than $40 \mathrm{MeV} /$ nucleon $^{38}$, why does this acceleration seem more efficient for certain species than others, and how are these ions transported inward of Io's orbit? At least seven more non-proton ion species at energies well into the $\mathrm{MeV}$ range have been reported at Jupiter ( $\mathrm{He}, \mathrm{C}, \mathrm{N}, \mathrm{Na}, \mathrm{Mg}, \mathrm{Si}, \mathrm{Fe}$ ), concentrated in either the extended magnetosphere, the radiation belts, or in both regions. The diverse observable "zoology" of ions that are rare at other planets makes Jupiter the best site within the accessible universe to study ion acceleration through leveraging its mass and charge-dependence ${ }^{39}$. In addition, elucidating the origin of Jupiter's energetic heavy ions will foster our understanding of the intense X-Ray aurora emitted by the interaction of these ions with the giant planet atmosphere ${ }^{40}$.

Missing in situ measurements: As for electrons and protons, the radiation belts need to be explored with direction, energy, and species resolved observations of heavy ions at energies reaching at least up to $1 \mathrm{GeV} / \mathrm{n}$. In addition, the charge state of heavy ions has only been inferred indirectly in the past and now needs to be directly measured. X-Ray emissions generated by the interaction of heavy ions with the Jovian atmosphere and the Io torus should also be monitored.

\section{4) Is high latitude charged particle acceleration a significant radiation belt source?}

Recent discoveries by the NASA's polar orbiting Juno mission have put the spotlight on highlatitude auroral processes as a means to generate high-energy charged particles, with in particular the prevalence of intense upward energetic $(>0.1$ to $>10 \mathrm{MeV})$ particle beams ${ }^{41,42,43}$. A certain fraction of these field-aligned particles may have their traveling direction scattered enough to become trapped, thereby populate the middle 44 or even the edge of the magnetosphere. Subsequent radial transport may ultimately feed the inner core of the radiation belts (see Figure 2). Similar hypotheses have been put forward at Earth ${ }^{45}$ and Saturn ${ }^{46}$, but the fraction of auroral particles that can supply the radiation belts is unknown. Still, the auroral source is so intense that, if $0.1 \%$ of particles become trapped, it could repopulate the entire 
radiation belts of Jupiter in 40 days ${ }^{47}$. This hypothesis therefore deserves to be explored.

Figure 2. The concept of high-latitude electron sources evolving into radiation belt sources. Courtesy: George Clark.

Missing in situ measurements: The link between auroral and equatorial energetic particles will be advanced by comparing Juno's polar observations with those gathered near the equator by Galileo, JUICE and

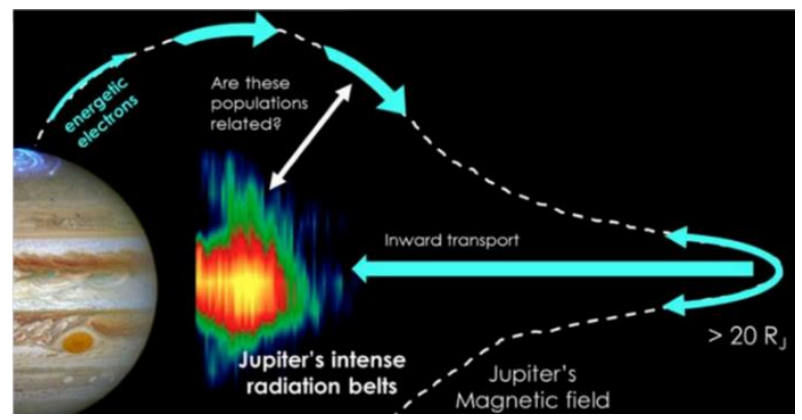
Europa-Clipper. However, spectral information with sufficient fidelity will only extend up to a few MeV. Energy-integrated measurements are insufficient given that auroral processes often lead to narrow peaks in the energy distribution. Energy-resolved measurements extending to energies higher than $1 \mathrm{MeV}$ would allow us to compare if the belt's seed population shares common properties with the high latitude accelerated products. In addition, concurrent observations of plasma, magnetic and electric fields, and electrostatic and electromagnetic waves are needed to reveal the acceleration and scattering mechanisms. Both high latitudes low altitudes and distant equatorial regions should be explored in situ.

\section{5) What is the role of geologically active moons in planetary radiation belts? The case of Io.}

Io is tiny compared to Jupiter, yet it controls most aspects of its magnetosphere ${ }^{25,48}$ and has a special and enigmatic role in shaping the most hazardous radiation belts of our solar system, as it appears to be both a source and a sink for them. The eruptions of hundreds of volcanoes source the moon's atmosphere, which in turns creates a dense neutral torus near its orbit. While high ionization rates prevent the Io-generated neutral torus from directly extending far from Io, sputtering of the surface of Europa by energetic charged particles produces another neutral torus which extends the neutral tori region outward of Europa's orbit ${ }^{63}$. Dissociation and ionization of Io neutral torus particles is the main source of cold plasma in Jupiter's magnetosphere ${ }^{48}$ and thus provides a dominant seed population for the radiation belts. The acceleration of freshly ionized material by the corotation electric field of Jupiter creates the most intense ElectroMagnetic Ion Cyclotron (EMIC) waves ever detected in planetary radiation belts ${ }^{49}$. Whistler-mode hiss and chorus waves have been detected near the moon's orbit ${ }^{50,51}$, and auroral acceleration processes have been observed in the flux tube connected to Io ${ }^{52}$.

The direct region of Io's influence extends from roughly $\mathrm{L}=5$ to $\mathrm{L}=7$. While small compared to the overall size of Jupiter's magnetosphere, this region encompasses almost all radiation belt processes at work in the magnetosphere ${ }^{36,38,50,52,53}$ : adiabatic radial transport (question 1), acceleration and losses caused by wave-particle interaction for electrons and ions (questions 1 and 3), auroral acceleration (question 4), absorption of electrons and ions by Io itself, sputtering of the moon's atmosphere (question 6) and loss of ions by charge exchange with the neutral torus. On top of that, the possible dawn-to-dusk electric field in the Io plasma torus may control the radiation belt time variability ${ }^{54}$ (question 7). The vicinity of Io's drift shell is therefore a test bed of choice for radiation belt physics that is contained within the enormous magnetospheric laboratory, and its smaller size facilitates its focused exploration.

Missing in situ measurements: All measurements that are required to answer other questions are needed from $\mathrm{L}=5$ to $\mathrm{L}=7$ at all latitudes in order to disentangle the processes at work in Io's vicinity and significantly advance radiation belt physics. 
6) How are moon surface material and possible biosignatures processed by radiation?

All the moons that orbit within the magnetosphere of Jupiter are weathered by the precipitation of energetic charged particles trapped in the radiation belts, making them invaluable case studies to compare with observations at other satellites across the Solar System. (1) Energetic ions, typically $>1-100 \mathrm{keV}$, are significant contributors to the sputtering of surface material and the generation of the atmospheres of Europa ${ }^{55}$, Ganymede ${ }^{56,57}$, and Callisto ${ }^{58}$. (2) The intrinsic magnetic field of Ganymede challenges our understanding of the moon's surface weathering. Models predict that Ganymede's equatorial regions are shielded against low energy particles as only tens of $\mathrm{MeV}$ electrons ${ }^{59}$ and $\sim 100 \mathrm{keV}$ ions ${ }^{57}$ can access and alter them, as is shown by Figure 3 for electrons. Different fluxes to Ganymede's polar and equatorial surface are thought to be responsible for the formation of Ganymede's bright polar caps and dark equatorial regions $^{60}$. (3) In addition, energetic electrons and ions have penetration depths from centimeters to several meters of depth ${ }^{13}$ and may alter the material crystallinity and thermal inertia or may create new molecules by radiolysis ${ }^{11}$ within these layers. (4) Finally, radiation belt particles which can have GCR-like energies at Jupiter may control the production of cosmogenic nuclides on the icy moons, the abundance of which can be used to date their surfaces ${ }^{74}$.

Figure 3 Surface map of $<30 \mathrm{MeV}$ electrons precipitating onto Ganymede's surface. The white color indicates a number flux equals to 0. Around 40\% of Ganymede's surface is completely shielded against $<30$ MeV electrons. Courtesy: Lucas Liuzzo ${ }^{59}$

These modifications must be considered

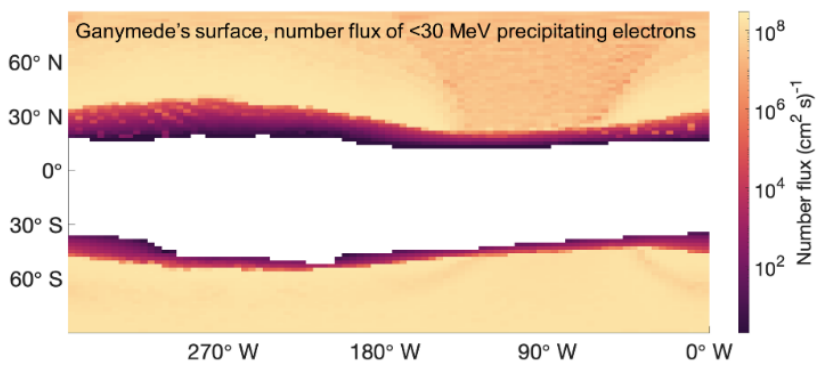
when interpreting remote sensing observations of the moons (e.g., reflectance spectra). For Europa, where future orbiters and landers will seek biosignatures in material that may have upwelled from its subsurface ocean, the study of radiation-induced surface modifications is crucial to understand how radiation limits life $e^{13}$ or conversely sustains it ${ }^{61,62}$. Finally, the composition and sheer intensity of the innermost radiation belt environment make it a unique laboratory to study the interaction of energetic plasma with the four airless moons Metis, Adrastea, Amalthea, and Thebe.

Quantifying any of the mechanisms discussed above depends considerably on the input energetic particle spectra to which a surface is exposed. Energy spectra are poorly constrained for $>1 \mathrm{MeV}$ electrons and $>10 \mathrm{MeV} /$ nucleon ions, so that studies which focus on the equatorial regions of Ganymede or on deeply penetrating particles at any moon rely so far on highly uncertain extrapolations to energies of $100 \mathrm{~s}$ of $\mathrm{MeV}$.

Missing in situ measurements: The energy range that should be covered by in situ charged particle measurements is similar to those discussed in sections 1 to 6 , with direction and energy resolved observations of electrons and ions up to $100 \mathrm{~s}$ of $\mathrm{MeV} /$ nucleon. Understanding if certain physical processes, like CRAND (question 2), contribute to the radiation belt content would allow us to physically extrapolate measurements to even higher energies.

\section{7) How and why do Jupiter's radiation belts change over time? (Space Weather)}

The intensity of the synchrotron radiation signal emitted close to Jupiter by trapped electrons is seen from Earth to change over time by a factor of $\sim 2$ to 3 on timescales that track the solar cycle $^{64,65}$, or on shorter timescales of weeks, days or hours ${ }^{66,67}$. Long-term synchrotron radiation observations from inside the radiation belts are ongoing with Juno ${ }^{68}$. However, synchrotron observations are integrated over the observer line of sight and a large energy range, so that direction and energy resolved in situ measurements close to Jupiter in the 1-10s MeV energy 
range are needed to interpret the observable variability in synchrotron emissions: do they reveal a change in the flux intensities, energy spectral slope and/or a redistribution of electrons along and across magnetic field lines? The Galileo orbiter, which orbited Jupiter for eight years, observed further away and at lower energies than synchrotron observations, that in situ electron and ion flux intensities vary over time by a factor of $\sim 10-20^{69}$. In addition, exceptional transient enhancements of very energetic $>11 \mathrm{MeV}$ electron fluxes have been detected, for instance during Galileo's orbit 22 near Ganymede's L shell ${ }^{33}$. It is important to understand the observed time variabilities for three reasons: (1) different radiation belt physical processes that may be difficult to distinguish in average profiles may be resolved through their distinct variability timescales; (2) to advance the understanding of radiation impact on moons, such as the generation of transient exospheres ${ }^{70}$ and; (3) to anticipate the radiation hazard posed to artificial satellites. For instance, the study of the long-term modulation of energetic protons at Saturn helped to estimate the contribution of the relatively constant CRAND source ${ }^{71}$.

Various inputs and mechanisms have been proposed to explain the time variability of the Jovian radiation belts. Cometary impacts are known to modify the innermost radiation belts ${ }^{66,72}$. The solar UV/EUV irradiance may change the radial diffusion of trapped particles, by modulating the speed of neutral winds in Jupiter's ionosphere and subsequent large-scale electromagnetic perturbations ${ }^{67}$. Changes in the possible dawn-dusk electric field in the Io plasma torus could also be a driver ${ }^{54}$. Finally, the upstream solar wind is likely to affect the Jovian radiation belts, even if the chain of processes needed to transmit its effects down to the innermost part of the gigantic magnetosphere remains a mystery. A solar wind-driven modification of the magnetospheric reservoir, maybe partly sourced by polar auroral activity, can be envisioned as a plausible mechanism.

Missing measurements: Any measurement conducted to answer questions 1 to 6 over extended periods of time would help to answer this question. Everywhere in the radiation belts, different time scales can be probed in situ by regulating the resampling rate, as done at Saturn with Cassini ${ }^{17,73}$. The time variability of the radiation belts of Jupiter is a long-standing open science question that has been debated for at least four decades. Advancing on it requires to combine together as many inputs and observations as possible: solar wind measurements just upstream of Jupiter, observation of the belt's seed population outward of Europa's orbit with Energetic Neutral Atoms and/or X-ray imaging, remote observation of the Io torus UV brightness, radio observations of the electron synchrotron radiation, and in situ electron and ion data.

References (* means JGR: Space Physics, ' means Icarus): ${ }^{1}$ Turner+, A\&A $2019{ }^{2}$ Shprits,$+ * 2012{ }^{3}$ Mauk \& Fox, * $2010{ }^{4}$ Mauk, * $2014{ }^{5}$ Lejosne \& Kollmann, SSR $2020{ }^{6}$ Dialynas+, * $2013{ }^{7}$ OPAG, OPAG Report 2019 ${ }^{8}$ Kollmann+, GRL $2016{ }^{9}$ Kollmann+, GRL $2018{ }^{10}$ Nénon \& André, GRL $2019{ }^{11}$ Paranicas+, ' $2018{ }^{12}$ Plainaki+, SSR $2018{ }^{13}$ Nordheim+, Nat. Ast. $2018{ }^{14}$ Drake \& Hvatum, Astr. Journ. $1959{ }^{15}$ Alfonsi+, Phys. \& Chem. 2017 ${ }^{16} \mathrm{Wu}+$, Adv. Sp. Res. $2019{ }^{17}$ Roussos, Kollmann+, Science $2018{ }^{18}$ Ripoll+, * $2020{ }^{19}$ Kollmann+, GRL $2017{ }^{20}$ Becker+, GRL $2017{ }^{21}$ Williams+, ApJ $2015{ }^{22}$ Williams+, AAS $2018{ }^{23}$ Kao, Pineda+, Astro2020 White Paper $2020{ }^{24}$ NRC, Vision and Voyages $2011{ }^{25}$ Keane+, 2023-2032 Planet. \& Astrobio. Dec. Surv. White Paper 2020 ${ }^{26}$ Fischer+, Science $1996{ }^{27}$ Horne+, Nat. Phys. $2008{ }^{28}$ Woodfield+, $* 2014{ }^{29}$ de Soria-Santacruz+, $* 2017{ }^{30}$ Thorne+, Nature $2013{ }^{31}$ Reeves+, Science $2013{ }^{32}$ Woodfield+, GRL $2019{ }^{33}$ Roussos+, ‘ $2018{ }^{34}$ Selesnick+, * $2013{ }^{35}$ Kollmann+, ' $2013{ }^{36}$ Nénon+, * $2018^{37}$ Allen+, GRL $2019{ }^{38}$ Garrard+, Science $1996{ }^{39}$ Kronberg+, * $2019{ }^{40}$ Gladstone+, Nature $2002{ }^{41}$ Paranicas+, * $2018^{42}$ Mauk+, GRL $2017{ }^{43}$ Allegrini+, * $2020{ }^{44}$ Kollmann+, * $2018{ }^{45}$ Fritz, SSR $2001{ }^{46}$ Roussos,$+{ }^{\prime} 2016{ }^{47}$ Mauk, AGU Fall Meeting $2019{ }^{48}$ Bagenal \& Dols, * $2020{ }^{49}$ Blanco-Cano+, * $2001{ }^{50}$ Nénon+, * $2017{ }^{51}$ Shprits+, Nat. Comm. $2018{ }^{52}$ Szalay+, * $2020{ }^{53}$ Paranicas+, GRL $2019{ }^{54} \mathrm{Han+}, * 2018{ }^{55}$ Cassidy+, ‘ $2013{ }^{56}$ Marconi, ‘ $2007{ }^{57}$ Poppe+, * $2018{ }^{58}$ Vorburger+, ' $2015{ }^{59}$ Liuzzo+, * $2020{ }^{60}$ Khurana+, ' $2007{ }^{61}$ Chyba, Nature $2000{ }^{62}$ Cooper+, ' $2001{ }^{63}$ Smith+, ApJ $2019{ }^{64}$ de Pater and Dunn, ' $2003{ }^{65}$ Santos-Costa+, * $2008{ }^{66}$ Santos-Costa+, * $2011{ }^{67}$ Kita+, * $2015{ }^{68}$ Santos-Costa+, GRL $2017{ }^{69}$ Jun+, ' $2005{ }^{70}$ Millilo+, PSS $2016{ }^{71}$ Kollmann+, Nat. Astr. $2017{ }^{72}$ Brecht+, ' $2001{ }^{73}$ Roussos+, PSS $2014{ }^{74}$ Hedman, ' 2019 